\title{
Aiming at Optimal Physical Activity for Longevity (OPAL)
}

\author{
Martin Kopp and Martin Burtscher ${ }^{*}$ (]
}

\begin{abstract}
Although even small volumes (15-20 min) of daily physical activity (PA) are associated with health benefits, the optimal volume for healthy aging and longevity is substantially larger, amounting to about 100 min of daily moderate PA. The accomplishment of this objective likely requires the development of an appropriate PA lifestyle at an early age. Research initiatives are necessary addressing the motivational contribution of families, school surroundings and sport clubs, perhaps combined with role model effects and instructions for the specific implementation. Such approaches would need an enlarged agreement in readdressing this new aim followed by the launch of a new research strategy in order to develop specific offers for the respective age cohorts.
\end{abstract}

Keywords: Exercise, Fitness, Health, Age, Motivation

\section{Key Points}

- Approaches to induce optimal rather than minimal amounts of physical activity are lacking.

- One possible approach to achieve optimal amounts of physical activity could be the foundation for high fitness levels and adherence to lifelong physical activity at an early age.

\section{Minimal Versus Optimal Physical Activity Recommendations}

Regular physical activity (PA) represents the most important lifestyle component associated with cardiorespiratory fitness, healthy aging and longevity. In order to induce general health benefits, public health guidelines recommend, beside strength and balance exercises, at least $150-300 \mathrm{~min}$ of PA at moderate aerobic intensity or $75-150 \mathrm{~min}$ at vigorous intensity per week $[1,2]$. Much more engagement in PA, however, is necessary to achieve maximal benefits on longevity [3]. For instance,

*Correspondence: martin.burtscher@uibk.ac.at

Department of Sport Science, University of Innsbruck, Fürstenweg 185, 6020 Innsbruck, Austria a large prospective cohort study demonstrated maximal longevity gains at about $700 \mathrm{~min}$ of moderate or 350 min of vigorous PA per week [4]. The accomplishment of this objective is challenging in particular when considering that even minimal PA recommendations are difficult to achieve in the aging population [5]. Comprehensive programs have been developed to support older adults in reaching these minimal recommendations, e.g. through providing them with timely motivational messages and real-time feedback by smartphones and smartwatches [5]. In contrast, the evaluation of approaches intended to induce and maintain optimal rather than minimal PA recommendations is lacking but would be of utmost importance with regard to healthy aging and longevity. Such approaches likely require early adoption and maintenance of appropriate types and amounts of regular PA. For instance, lifelong aerobic exercise will not only impact on longevity but will also slow down the aging-related decline of general fitness. Remarkably higher cardiovascular and skeletal muscle metabolic fitness have been demonstrated among lifelong male and female exercisers in their 8th decade of life when compared to healthy non-exercisers [6]. The associated large physiological reserve above the frailty threshold supports 
not only individual independence but also participation in social life.

\section{Do Physical Activity Levels During Childhood and Adolescence Track into Adulthood?}

From physiological as well as psychological perspectives, it makes sense to lay the foundation for high fitness levels and adherence to lifelong PA at an early age. A prerequisite, however, is that PA levels during childhood and adolescence track into adulthood. If this holds true, parents and physical education teachers could beneficially and importantly affect healthy aging and longevity. This has actually been demonstrated by several studies, indicating that PA lifestyle starts to develop in early childhood and that certain types of PA, e.g., running, and the consistency of participation during adolescence are associated with PA levels in adulthood [7-10]. Very recently, a randomized trial protocol has been presented examining motivational, regulatory, and habitual intervention approaches focusing on parent-child activities together [11]. The need of such interventions is underlined by study findings including monozygotic twins indicating beneficial long-term results by targeting childhood and adolescent environments like families and schools [12].

\section{Is Motivation the Key?}

Motivation represents undoubtedly a critical factor for sustained exercise, with intrinsic motivation being especially important for long-term adherence [13]. Available data suggest that individuals with a high exercise adherence report positive exercise experiences including enjoyment in the past. Consequently, considerable importance is attached to the motivation by appropriate offers and continuation of PA and sports during childhood and youth [14]. Although motivational theories have considerably contributed to the increase of health enhancing PA, it will be a step forward to evaluate these theories in their efficacy on an optimal level of PA related to longevity (OPAL). Therefore, research initiatives addressing the motivational contribution of families, school surroundings and sport clubs, perhaps combined with role model effects and instructions for specific implementation, might be a promising path. The results will launch an educational gateway to sport and exercise, which may enable a lifelong flow between different motives to ensure OPAL, including health, social as well as performance related motives.

\section{Conclusions and Suggestions for Implementation}

Although the transfer of recommended PA in childhood over the entire life span constitutes a target that is easy to understand and communicate, it requires joint and continuous efforts of decision-makers, including families, schools, sport clubs, healthcare providers, and policy-makers. First, easy access to child- and youthoriented sports and PA possibilities, adapted to local conditions, constitutes a prerequisite. Further, a special focus must be set on the critical phase of the transition from school to working life, e.g., by specific sport offerings, including participation in sports groups, access to fitness studios, encouragement for participation in sporting events, etc. Moreover, the promotion of parent-child activities, appropriate sport and PA offerings for elderly individuals, but also disabled persons will help to prevent interruption of usual PA. These suggestions may not sound very revolutionary; however, convincing overall concepts and appropriate regimes for the control of their implementation are largely lacking.

Beside sport offerings, an abundance of evidencebased measures has been proved to effectively support PA behavior in various age and community groups. For instance, supporting the reduction of sitting and screen times will help to achieve higher PA levels, being of particular importance for adolescents [15]. Appropriate measures include a combination of motivational/ volitional (e.g., health promotion by mass media), environmental (e.g., use of sit-stand or standing desks) and policy (e.g., curriculum change) strategies [16]. Promotion to increase every day physical activity like stair climbing was demonstrated to improve several health attributes, e.g., increased cardio-respiratory fitness, reduced risk of falling, and less perceived strain during daily life activities, particularly in the elderly [17]. Moreover, active commuting $[18,19]$ and gamification of PA constitute further promising applications to support PA behavior in healthy and diseased people as well [20]. Finally, societal changes such as city architecture and urban planning [21] or financial incentives [22] may effectively support the increase of PA and/or the improvement of adherence to PA recommendations.

The distinction between performance-related and health-related PA practice would have to be rethought and decision-makers would have to work systematically on an interaction between performance goals, social goals and health goals, backed with a metaplan for OPAL. Of course, this approach would need an enlarged agreement in readdressing this aim followed by the launch of a new research strategy in order to develop specific options for the respective age cohorts.

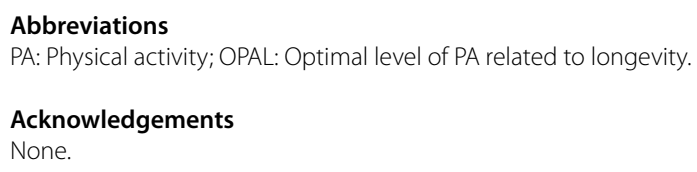




\section{Declarations}

\section{Authors' Contributions}

MK and MB contributed equally to the article, read and approved the final manuscript.

\section{Funding}

No sources of funding were used in the preparation of this article.

\section{Availability of Data and Materials}

Not applicable.

Ethical Approval and Consent to Participate

Not applicable.

\section{Consent for Publication}

Not applicable.

\section{Competing interests}

Martin Kopp and Martin Burtscher declare they have no competing interests relevant to the content of this article.

Received: 4 March 2021 Accepted: 17 September 2021

Published online: 09 October 2021

\section{References}

1. Piercy KL, Troiano RP, Ballard RM, Carlson SA, Fulton JE, Galuska DA, et al. The physical activity guidelines for Americans. JAMA. 2018;320(19):2020-8.

2. Bull FC, Al-Ansari SS, Biddle S, Borodulin K, Buman MP, Cardon G, et al. World Health Organization 2020 guidelines on physical activity and sedentary behaviour. Br J Sports Med. 2020;54(24):1451-62.

3. Arem H, Moore SC, Patel A, Hartge P, Berrington de Gonzalez A, Visvanathan $\mathrm{K}$, et al. Leisure time physical activity and mortality: a detailed pooled analysis of the dose-response relationship. JAMA Intern Med. 2015;175(6):959-67.

4. Wen CP, Wai JP, Tsai MK, Yang YC, Cheng TY, Lee MC, et al. Minimum amount of physical activity for reduced mortality and extended life expectancy: a prospective cohort study. Lancet. 2011;378(9798):1244-53.

5. Boulton E, Hawley-Hague H, French DP, Mellone S, Zacchi A, Clemson L, et al. Implementing behaviour change theory and techniques to increase physical activity and prevent functional decline among adults aged 61-70: the PreventIT project. Prog Cardiovasc Dis. 2019;62(2):147-56.

6. Gries KJ, Raue U, Perkins RK, Lavin KM, Overstreet BS, D'Acquisto L, et al. Cardiovascular and skeletal muscle health with lifelong exercise. J Appl Physiol. 2018;125(5):1636-45.

7. Bélanger M, Sabiston CM, Barnett TA, O'Loughlin E, Ward S, Contreras G, et al. Number of years of participation in some, but not all, types of physical activity during adolescence predicts level of physical activity in adulthood: results from a 13-year study. Int J Behav Nutr Phys Act. 2015;12:76.

8. Craigie AM, Lake AA, Kelly SA, Adamson AJ, Mathers JC. Tracking of obesity-related behaviours from childhood to adulthood: a systematic review. Maturitas. 2011;70(3):266-84.
9. Telama R. Tracking of physical activity from childhood to adulthood: a review. Obes Facts. 2009;2(3):187-95.

10. Telama R, Yang X, Leskinen E, Kankaanpää A, Hirvensalo M, Tammelin T, et al. Tracking of physical activity from early childhood through youth into adulthood. Med Sci Sports Exerc. 2014;46(5):955-62.

11. Grant SJ, Beauchamp MR, Blanchard CM, Carson V, Gardner B, Warburton DER, et al. Parents and children active together: a randomized trial protocol examining motivational, regulatory, and habitual intervention approaches. BMC Public Health. 2020;20(1):1436.

12. Simonen RL, Videman T, Kaprio J, Levälahti E, Battié MC. Factors associated with exercise lifestyle: a study of monozygotic twins. Int J Sports Med. 2003;24(7):499-505

13. Teixeira PJ, Carraça EV, Markland D, Silva MN, Ryan RM. Exercise, physical activity, and self-determination theory: a systematic review. Int J Behav Nutr Phys Act. 2012;9:78.

14. Rodrigues F, Teixeira DS, Neiva HP, Cid L, Monteiro D. Understanding exercise adherence: the predictability of past experience and motivational determinants. Brain Sci. 2020;10(2):98

15. Hardy LL, Ding D, Peralta LR, Mihrshahi S, Merom D. Association between sitting, screen time, fitness domains, and fundamental motor skills in children aged 5-16 years: cross-sectional population study. J Phys Act Health. 2018;15(12):933-40.

16. Nguyen P, Le LK, Nguyen D, Gao L, Dunstan DW, Moodie M. The effectiveness of sedentary behaviour interventions on sitting time and screen time in children and adults: an umbrella review of systematic reviews. Int J Behav Nutr Phys Act. 2020;17(1):117.

17. Donath L, Faude $O$, Roth R, Zahner L. Effects of stair-climbing on balance, gait, strength, resting heart rate, and submaximal endurance in healthy seniors. Scand J Med Sci Sports. 2014;24(2):e93-101.

18. Celis-Morales CA, Lyall DM, Welsh P, Anderson J, Steell L, Guo Y, et al. Association between active commuting and incident cardiovascular disease, cancer, and mortality: prospective cohort study. BMJ. 2017;357:j1456.

19. Reich B, Niederseer D, Loidl M, Fernandez La Puente de Battre MD, Rossi VA, Zagel B, et al. Effects of active commuting on cardiovascular risk factors: GISMO-a randomized controlled feasibility study. Scand J Med Sci Sports. 2020;30(Suppl 1):15-23.

20. Agarwal AK, Waddell KJ, Small DS, Evans C, Harrington TO, Djaraher $R$, et al. Effect of gamification with and without financial incentives to increase physical activity among veterans classified as having obesity or overweight: a randomized clinical trial. JAMA Netw Open. 2021:4(7):e2116256.

21. Sallis JF, Cerin E, Conway TL, Adams MA, Frank LD, Pratt M, et al. Physical activity in relation to urban environments in 14 cities worldwide: a crosssectional study. Lancet. 2016:387(10034):2207-17.

22. Barte JCM, Wendel-Vos GCW. A systematic review of financial incentives for physical activity: the effects on physical activity and related outcomes. Behav Med. 2017:43(2):79-90.

\section{Publisher's Note}

Springer Nature remains neutral with regard to jurisdictional claims in published maps and institutional affiliations.

\section{Submit your manuscript to a SpringerOpen ${ }^{\circ}$ journal and benefit from:}

- Convenient online submission

- Rigorous peer review

- Open access: articles freely available online

- High visibility within the field

Retaining the copyright to your article

Submit your next manuscript at springeropen.com 